\title{
Narrative Style Centered on Humanitarian Spirit in Folk Narrative of China Contemporary Photography
}

\author{
Bing Hao \\ Henan University Minsheng College \\ Kaifeng, China 475004
}

\begin{abstract}
This article discusses the theme of humanitarian spirit in folk narrative features of China contemporary photography and specifically explains the narrative style centered on humanitarian spirit in China contemporary photography, namely, the exploration of the deep narrative space and the unique individual narrative language. In general, shooting the things that are needed to narrate can realize the narrative. What we care about is how the folk narrative in Chinese contemporary photography expresses the theme of humanitarian spirit, that is, how the folk narrative of contemporary photography concentrating on humanitarian spirit narrates the story.
\end{abstract}

Keywords - Chinese contemporary photography; folk narrative; humanistic spirit; narrative way

\section{INTRODUCTION}

As a typical narrative work, photographic work has got more and more attention from the narrative theory. Photography uses camera lens to narrate stories and the lens is a pair of eyes, which is a kind of imperceptible language that is different from the text.

The theme of Chinese humanitarian spirit is an abroad and open concept, which involves the compassion for the insulted and injured people and the protection of humanity and the liberation of individuality. This article focuses on photography, because the twenty-first century is the era of reading pictures. Images become more and more important in people's daily life and have more intuition in the representation on the theme of humanitarian spirit.

The narrative way is how to narrate, that is, how to unfold the story and narrate the story through the language, and the prominent point is "narration". The image has narrative power. Someone runs and takes pictures. The pictures tell the reader that someone is running and that's the narrative. Using images to narrate is the most important function for pictures. Compared with other forms of art, it is the most unique function. In general, shooting the things that are needed to narrate can realize the narrative. What we care about is how the folk narrative in Chinese contemporary photography expresses the theme of humanitarian spirit, that is, how the folk narrative of contemporary photography concentrating on humanitarian spirit narrates the story.

\section{THE EXPLORATION OF DEEP NARRATIVE SPACE}

Chinese contemporary folk narrative photography expresses a profound sense of space and the applied art language mainly includes line and tone. The expression of pace mainly includes two ways: physical perspective and air perspective.

\section{A. Physical Perspective Is Also Linear Perspective}

The influence of camera site on the perspective, that is, the expression of the depth of space is determined by the distance of the camera from the target and the change of the shooting point. Folk narrators often apply the way of overlooking to enhance the line perspective of plane scenery and render the emotions, creating an emotional sense of oppression. In twentieth century, with the reform and opening up, a large number of farmers entered the city and started their new life, but the peasants remained in a state of opposition to the city and urban life in terms of psychology and real life. Zhang Xinmin's image project, Encompass the City-the Long March of Chinese Peasants to the City, which describes the whole process of Chinese peasants flowing from the countryside to the city and surviving in the city. It presents the most significant events in the changing of contemporary Chinese society in a panoramic scale: the process of farmers leaving the land and integrating into the city. The picture of Go into the City presents a window cleaner in the high-rise, which typically shows the opposition. He shot a migrant worker working outside the window from a downward angle which causes strong perspective relations. We can find the worker in the picture with his surrounding cement jungle forms a kind of sinister relationship that people live in the city as if on the brink of a deep gulf, and this is the most eloquent way of visually defining the farmer's place in the city. The surrounding environment is indifferent to him and he is helpless in this city space. The helpful resources are limited to him, just like the rope in his hand, and he is isolated and helpless in this city.

Folk narrative photography mostly adopts wide-angle lens, and wide-angle lens has a large depth of field and a strong perspective effect, which is beneficial to show depth space effect. Wide-angle lens shoots the same scene picture can not only highlight the main characters, but also show the characteristics of environment. Therefore, with a wide-angle lens to shoot the close shot of figure, it can not only see people's facial expression, but also explain the environment 
where people stay in, so as to achieve the purpose of environmental contrasting characters and the explanation of event content, at the same time, the explanation of relationship between characters and their environment can increase the authenticity of events.

\section{B. Air Perspective Is Also Tone Perspective}

Folk narrative photographers often use rich tone level to guide people's attention to the depth of painting and put the viewer's imagination into the deep space.

The Goose Farm is a beautiful picture of the socialist countryside with a clear conception, a vivid theme and a rich flavor of life. The application of light, the processing of idea, the allocation of chiaroscuro and tone and the grasp of moment can be the first-rate. Through a corner of goose farm, the sunny backlighting and the foreground locust tree, the numerous strong beam of light illuminates the whole picture, forming a clear atmosphere and a harmonious warm tone (In the back of locust tree, burning some weeds and triggering a smoke, through the illuminating of backlighting, a lot of sunshine has produced through the gap a tall tree branches.); through the application of backlighting, the foreground, medium shot and panorama has become hustle and bustle; the spread gooseneck like bamboo and forest produces the contrast of light and shade in the far and near and the size, showing a strong sense of space.

In a word, folk narrative photography controls the space and depth of the scene by means of photography molding technique, reflecting the original living condition of the ordinary people in china.

\section{INDIVIDUAL NARRATIVE LANGUAGE WITH UNIQUE CHARACTERISTICS}

The life experience and artistic accomplishment are different from person to person, so the ways of expression are different. The best way to express them becomes the language he holds. Based on the characteristics of folk narrative photography, we believe that folk narrative photography and narrative language belong to the author's personal visual language. It also applies light, tone, lines and colors to constitute their modeling language.

\section{A. The Visual Language of Photographic Properties}

Folk narrative photography applies light, tone, lines to constitute their modeling language and photographers build up the beauty of photography with these languages.

\section{1) Lines}

Our word is full of lines and different lines evoke different emotional responses. Folk narrative photographers pay great attention to the application of lines. They not only apply the beauty of lines to construct and enrich images, but also regard lines as living things, so as to express their emotional and enhance their artistic appeal. The Waltz of Baosteel is a musical composition composed of lines and notes. The author says "I try to demonstrate the characteristics of modern industry by applying the rhyme and rhythm of the lines." On the picture, the silver gray plate of the curved line is surrounded by layers after layers, scattered orderly, from the near to the distant, from large to small, from height to low, from light to dark, from the real to imaginary, from transverse to vertical and from objectives to people... constitutes a gradient of rhythm, like a soft, vivid, beautiful and moving movement. The organic combination of lines and tone makes the screen unified and active, neat but not dull, which makes people realize the rhyme and rhythm with formal beauty.

Shape is the most basic element in composition of a picture. A square and a rectangle are enclosed by four straight lines and this shape gives a sense of placidity and stability. $\mathrm{Hu}$ Wugong's Square Shaped City has shown us a sense of placidity and stability. Square Shaped City is an outward manifestation of the pursuit of a stable agricultural civilization mentality and $\mathrm{Hu}$ Wugong thinks that Square Shaped City is a symbol of ancient China society and history. He regards Xi'an as "Square Shaped City", the first is for its shape and the second is for coordinating with social content. Without bright colors and elaborate composition of picture, Hu Wugong's work is a kind of simple narration and the square composition of picture shows us the comfortable and steady living conditions of Xi'an people.

The line is the basic conditions of visual image and folk narrative photographers pay attention to the application of lines, and they not only apply the beauty of lines to construct and enrich images, but also regard lines as living things, so as to express their emotional and enhance their artistic appeal.

\section{2) Light}

Light is the soul and life of photography. Folk narrative photographers make full use of the characteristics of light to show the living conditions and behavior of ordinary people.

Light can act on the tone, line and color, highlight the body image, and enhance environmental atmosphere and the ability to shape the performance. Light can also act on people's thoughts and feelings and arouse people's different emotional associations and artistically express the specific affection, emotion and sentiment. In the process of artistic creation of folk narrative photography, many photographers pay great attention to the use of light to represent the specific mood, emotional appeal and changes in the picture. For example, they often apply the dim light and appropriate light and shade contrast to represent the miserable and solemn and respectful atmosphere in the scene of tragedy, and usually use soft and vivid lines to show the relaxed and pleasant emotion in the lively scene.

As folk narrative photographers have paid attention to the identity of sentiment and the use of light and the identity of sentiment change and light change in the whole picture, therefore, they begin to enhance the ideological level and the aesthetic meaning of the works.

The art of photography is the modeling art of "light" and "shadow". The process of photography completion must rely on light and correctly utilize and exert the function of light in photographic art modeling to record, reflect, express and deliver our creative thinking, emotion and performance techniques, so as to reach to the visual images of the picture. 


\section{3) Tone}

The tone refers to a general light and shade feature which constitutes the picture and is a series of different grades expression of black, white and gray. The tone is an important means to deal with figures and object modeling, to foil atmosphere and to express the feelings in photography. Different tone has different emotional colors.

Folk narrative photography that records the living state of ordinary people usually applies the middle tone to express the theme and the middle tone is composed of a variety of gray tone which is the main tone in photography. Because the main tone of middle tone is gray, the middle tone is also named gray tones. The middle tone is the basic tone of picture and can produce peaceful and remote feelings. Obviously, the middle tone lacks a strong impact force in tone, but it is relatively free in the aspect of performance. The picture closes to the life and is not swank and the subject is relatively strong, so folk narrative photography mainly uses the middle tone.

In folk narrative photography, the photographer's works comprehensively and profoundly reflect the rich and colorful life, which is often filled with sense of reality, dynamic and unique beauty of life. In the photographic works Miners of Song Chao, who deliberately enhances the file clarity and the details of the performance through a large format camera, with a white background and incomplete composition, and sets out the miner's black face and slightly numb state of mind after the mining work by contrast, with unique aesthetic pursuit to stock to people.

The tone level of folk narrative photography is very rich, however, this kind of tone, it can be said that it has specific emotional coloring and expresses various emotions.

\section{B. Photographer's Personal Visual Language}

Bao Kun said "the body language of photography refers to the derivative image features based on photography technology, and they are these basic things such as instantaneous sensation, sense of speed and tone etc." Obviously, what he refers to is the traditional photography language, that is, the so-called skills which is based on the basic technical level. As Bao Kun said "the obsolete language view makes all people say the same words and wear the same clothes, then there will be only one road that leads nowhere." However, the new concept of language is just to break all kinds of "conventions". As the folk documentary image of historical memory version, the choice of expression forms has laid down the keynote for the narrative content. When the records of social humanity are deeply explored, at the same time, it is often accompanied by the exploration of new themes, the formation of individual visual styles, the emergence of classical images and the publicity of emotions. Paying attention to different social problems, facing with different people, personal cut-in angle and way, the application of different image languages begin to differentiate and present the image forms with a strong subjective color and unique visual style. There are about four areas where using geographical features shows personalized photographic languages: Shanghai, Shanxi, Henan and Shandong. As the mainstay of Central Plain cultural tradition in Yellow River of
Chinese civilization, Henan photography shows the steady strength.

Based on the folk position, the language forms of many photographers no longer excessively pay attention to the mechanical changes of images and deliberately observe and study its relationship with linear structure. However, they integrate more personal thinking of the photographer and the image language becomes simpler, which is the author's personal visual language. Paying attention to different social problems, facing with different people, personal cut-in angle and way, the application of different image languages begin to differentiate and present the image forms with a strong subjective color and unique visual style.

\section{CONCLUSION}

In conclusion, folk narrative photography describes the living conditions of ordinary people by standing in the folk position through different narrative ways. It has the property of documentary photography and its own characteristics. It focuses on the folk, daily and various life details and goes deeply into the living of ordinary people to represent history from a perspective of folk, which aims to restore Chinese original life modality by the way of image. With rich, free and polymorphic personal viewpoint, it reflects the living condition of the ordinary people and provides a human scene with Chinese style to show the humanistic connotation of a great era

\section{REFERENCES}

[1] Luo Gang writes. Introduction to Narratology[M]. Kunming: Yunnan People's Publishing House, 1994.

[2] Zhang Qinghua writes. Evolution of Folk Concepts and Three Folklore Aesthetic Patterns in Contemporary Literature[J].Literature and Art Studies, 2002-2

[3] Mo Yan writes. Writing for the Common People[J]. Contemporary Writer Review, 2002-1.

[4] Gu Zheng writes. Between Reality and Imagination: Contemporary Photography and Urbanization in China[J] Image Era: The Theoretical Analysis of Visual Culture Communication, 2005-11.

[5] Shi Baoxiu writes. Image of the Times[M]. Guangzhou: Lingnan Fine Arts Publishing House, 2003.

[6] Yuan Yizhong writes. Master of Contemporary Photography: ---20 Witness of Human Nature[M]. Beijing: China Photographic Publishing House, 1988.

[7] Tang Dongping writes. Analysis of Photographic Works[M]. Hangzhou: Zhengjiang Photographic Press, 2006.

[8] Yang Enpu writes. Fundamentals of Photography Aesthetics[M]. Shenyang: Liaoning Fine Arts Publishing House, 1997.

[9] Lu Wei, An deming writes. The Diversity of Folk Narrative[M]. Beijing: Academy Press, 2006

[10] Li Qiang writes. Photographic Composition of Picture[M]. Guilin: Guangxi Normal University Press, 2004. 